\title{
The Medical Society for the study of Venereal Diseases, Spring Meeting 1996, Edinburgh 9-11 May
}

\section{Bone marrow as a diagnostic tool in HIV-positive patients with pyrexia of unknown origin}

G Brook ${ }^{1}$, H Ayles ${ }^{2}$, C Harrison ${ }^{3}$, C Rowntree ${ }^{3}$, RF Miller ${ }^{1}$. ${ }^{1}$ Mortimer Market Centre, ${ }^{2}$ Middlesex Hospital, ${ }^{3}$ University College Hospital, London

Objectives: To assess the effectiveness of bone marrow sampling in the diagnosis of the causes of pyrexia of unknown origin (PUO) in HIV-positive patients.

Methods: Patients who had undergone bone marrow sampling as one of the investigations for PUO between 1987 and 1995 were identified. From patient records and computer data the value of the marrow samples in achieving a diagnosis of the cause of the PUO was assessed.

Results: 117 patients had 121 marrow aspiration and 113 marrow trephine samples. Microscopic examination of the marrow revealed the cause of the pyrexia in 33 $(27 \%)$ of patients from $30(27 \%)$ trephine and $4(3 \%)$ aspiration samples. The diagnoses made were: mycobacterial infection (25), lymphoma (7) and toxoplasmosis (1). Factors more commonly found in patients with positive marrow samples than those with negative samples were low haemoglobin (mean $8.5 \mathrm{~g} / \mathrm{dl}$ vs $9.9 \mathrm{~g} / \mathrm{dl}$, p < 0.05 ), low CD4 count (mean 0.06 vs $0.102 \times 10^{9} / 1, \mathrm{p}<$ $0.05)$ and recent significant fall in any of the haemoglobin $(<8 \mathrm{~g} / \mathrm{dl})$, white cell $\left(<2 \times 10^{9} / \mathrm{l}\right)$ or platelet count $(<80$ $\left.\times 10^{9} / 1\right)(18 / 33$ [55\%] vs $27 / 88$ [31\%] of patients with diagnostic and non-diagnostic samples respectively, $\mathrm{p}<$ $0.05)$. When mycobacterial infection was diagnosed by microscopy of a marrow sample, this was achieved a mean (range) of 25 (8-60) days earlier than the first positive culture. Marrow trephine samples had a sensitivity of $25 / 39(64 \%)$ for the detection of mycobacterial infection. Conclusion: Marrow sampling, especially marrow trephines, contributed significantly to the early diagnosis of the cause of PUO. This success was due to the detection of mycobacterial infection, lymphoma and disseminated toxoplasmosis. Samples were most likely to be positive in patients with anaemia, low CD4 count or recent fall in platelet, red or white blood cell count.

\section{Investigation of a possible causal role for bacterial vaginosis (BV) in the development of non-gonococcal urethritis (NGU)}

FEA Keane ${ }^{1}$, B Thomas ${ }^{1}$, A Renton ${ }^{2}$, L Whittaker ${ }^{2}$, D Taylor-Robinson'. 'MRC STD Research Group, Jefferiss Wing, ${ }^{2}$ Academic Department of Public Health, Imperial College School of Medicine at St Mary's, London

Methods and results: Two studies were carried out in which NGU was diagnosed on the findings of $\geqslant 5$ polymorphonuclear leucocytes (PMNLs) per high-power field $(\mathrm{HPF})(\times 1000)$ in a Gram-stained urethral smear, or $\geqslant 10$ PMNLs per HPF in a Gram-stained thread obtained from a first-pass urine (FPU) sample. BV was diagnosed by examining a Gram-stained vaginal smear using Nugent's scoring system. Chlamydia trachomatis was detected by means of a direct immunofluorescent antibody test (MicroTrak; Syva) on urethral and FPU specimens from men and cervical and FPU specimens from women. In the first study (case controlled), involving 51 couples, 39 men with NGU were recruited, and 12 (31\%) of their female contacts had BV. In contrast, of 12 men without NGU, only $1(8 \%)$ of the female partners had BV (Fisher's exact test $\mathrm{p}=0 \cdot 15$; odds ratio $=4 \cdot 89$ ). When only $C$ trachomatis-negative patients were considered, the odds ratio for an association between $\mathrm{BV}$ and NGU was increased to $6 \cdot 77 ; p=0 \cdot 11$. The second (cohort) study ran concurrently with the first study and involved recruiting female patients with or without BV and examining their male partners for evidence of NGU. Thirty-eight couples were recruited. Of 17 women with BV, $12(71 \%)$ of their male partners had NGU. In contrast, of 21 women without BV, 7 (33\%) of their partners had NGU $(p=0 \cdot 049$; odds ratio $=4 \cdot 8)$. When only $C$ trachomatisnegative patients were considered, the significance was increased $(p=0.037$; odds ratio $=5 \cdot 42)$.

Conclusions: The evidence suggests that the endogenous bacteria of the vagina, when occurring in large numbers in BV, are capable of causing NGU in male partners.

\section{Is STD screening of semen donors necessary?}

JM Craig', CLR Barratt ${ }^{2}$, GR Kinghorn'. 'Department of GUM, Royal Hallamshire Hospital, ${ }^{2}$ University Reproductive Clinic, Jessop Hospital for Women, Sheffield

Aims: To evaluate need for semen donors STD screening.

Methods: Review of case notes of all potential semen donors referred by the University Reproductive Clinic for STD screening during the period 1 January 1992 to 31 December 1995.

Results: 173 of 175 men referred accepted screening; of whom, $97 \%$ were Caucasian and $75 \%$ were single. $49 \%$ were students, $42 \%$ employed and $9 \%$ were unemployed. Mean age was $26 \cdot 8$ years (SD $6 \cdot 1$ ). CMV seroprevalence was $26 \% .6 \%$ of donors had positive findings at their first screen; 4 with asymptomatic positive urethral HSV culture, 4 with asymptomatic NSU and 3 with urethral gp B streptococcal carriage. 109 donors (63\%) attended for rescreening after six months and/or on completion of semen donation. Reasons for non-reattendance included failure to donate or exclusion because of initial STD screen results, subsequent poor semen samples or on medical grounds. $12 \%$ had positive findings at second screening; 3 with genital warts, 1 chlamydial urethritis, 4 with NSU and 6 with urethral ureaplasma carriage. Positive findings at initial screening were predicted by a history of $>1$ partner in the preceding 6 months (OR 7.11, 95\% CI 
1.66-30-4). It did not predict re-screening findings. Other factors such as age, marital status, employment status or past STDs were not predictive for either screen.

Conclusions: The British Andrology Society recommends screening potential donors for sexually transmitted infections to minimise the risk of transmission of pathogens to the mother and foetus. At present, only $20 \%$ of potential donors meet the initial inclusion criteria of high quality semen with no transmissible genetic disorder or infection. Excluding all those with recent partner change would create difficulty in achieving adequate donor numbers and would not preclude all positive findings. Sequential STD screening of all semen donors is essential.

\section{High grade CIN: associations and screening strategies}

CJN Lacey, EF Monteiro, RIJ Macdermott, A Andrew, P Gibson. Departments of Genitourinary Medicine and Histopathology, General Infirmary, Leeds; Public Health Laboratory, London

Objectives: To investigate the epidemiologic, cytologic and virologic associations of CIN II/III and assess the performance of various screening strategies.

Design: A cross-sectional study of women, all of whom had epidemiological and sexual behaviour data recorded, a dot-blot hybridisation assay for HPV 6/11, 16, 18 and 31 performed, and colposcopy with biopsies of acetowhite areas carried out.

Setting: A genitourinary medicine clinic.

Subjects: 268 women with genital warts, 151 women with abnormal cytology and 51 control women.

Results: $8 \%$ of the control women, $35 \%$ of women with genital warts and $46 \%$ of the women with abnormal cytology had CIN II/III. In univariate analysis women with warts were younger, and women with abnormal cytology had more sexually transmitted disease and lifetime partners. In multivariate analysis only the presence of abnormal cytology (OR 3.0, 95\% CI 2.0-4.5 for mild dyskaryosis or worse) or HPV 16 (OR $3.8,95 \% \mathrm{CI}$ $2 \cdot 3-6 \cdot 3)$ were independently associated with CIN II/III. In this population a colposcopic referral policy of mild dyskaryosis or worse (200 colposcopies) discovered $62 \%$ of all CIN II/III and adding a positive HPV 16 test increased sensitivity to $72 \%$ ( 237 colposcopies). Using a positive test for HPV 16,18 or 31 to triage women with borderline and mild dyskaryosis (146 colposcopies) gave a sensitivity of $52 \%$.

Conclusions: This study confirms HPV 16 as the major independent risk factor for CIN II/III in a GU medicine clinic population. Further evaluation of reproducible oncogenic high risk HPV tests in cervical cancer screening and prevention is warranted.

\section{Oral candidal population dynamics in AIDS patients with recurrent oral candidosis}

C McCreary, D Bennett, D Sullivan, D Coleman, F Mulcahy. Department of Genitourinary Medicine, St James' Hospital, Dublin 8, Ireland

Objective: To investigate oral Candida population dynamics in a group of AIDS patients with a history of recurrent oral candidiasis following azole antifungal therapy.

Methods: Sixteen adult AIDS patients with a history of recurrent oral candidiasis, (12 IVDU, 4 homosexuals) were analysed for oral Candida species during each of 3 successive episodes of oral candidiasis. During each episode the patients were treated with azole antifungal drug (14 with fluconazole, 1 with itraconazole and 1 with both drugs). The relative abundance of each species present upon primary isolation was recorded during each episode. Persistence of Candida albicans strains was investigated by genomic DNA fingerprint analysis using the cloned $C$ albicans-specific repetitive sequence-containing DNA probe 27A.

Results: Five patients were infected with a single strain of $C$ albicans which persisted throughout the three episodes of infection, as determined by DNA fingerprint analysis. $C$ albicans was found to be the predominant species present during the initial episode of infection in 5 patients, but in each case this was completely replaced by a non-albicans species, either $C$ glabrata, $C$ krusei or the recently discovered species $C$ dubliniensis, by the second or third episode. In two patients, 2 non-albicans Candida species ( $C$ glabrata and either $C$ tropicalis or $C$ dubliniensis) were the only species recovered during the initial episode of infection; in one patient only one of these species ( $C$ dubliniensis) remained during the second and third episodes of infection. In the other patient only $C$ tropicalis was present by the third episode of disease along with $C$ albicans. Two of the remaining four patients harboured a non-albicans Candida species ( $C$ glabrata or $C$ tropicalis) in approximately equal abundance with $C$ albicans at the initial episode of infection; in one of these individuals $C$ albicans was the predominant species during the third episode. The converse was true for the other patients. The last two patients harboured a single strain of $C$ albicans at the initial episode of infection, which also persisted throughout the subsequent episodes, albeit in progressively lower abundance relative to $C$ glabrata.

Conclusions: Recurrent oral candidiasis in the group of patients studied was shown to be due to persistence of individual strains of the primary pathogen $C$ albicans and to be complicated by its displacement by non-albicans Candida species. This has important implications for the diagnosis and treatment of these highly susceptible patients.

\section{Association of antibodies to Chlamydia trachomatis heat-shock protein $60 \mathrm{kDa}$ with chronic non-gonococcal urethritis}

PJ Horner, M Ali, M McClure, D Cain, BJ Thomas, C Gilroy, D Taylor-Robinson. The Jefferiss Wing, Imperial College School of Medicine at St Mary's, London

Aims: To develop an indirect enzyme-linked immunosorbent assay (ELISA) using the heat shock protein, MW $60 \mathrm{kDa}$ (hsp60), of Chlamydia trachomatis as antigen and to use this to study the immunopathogenesis of chronic non-gonococcal urethritis (NGU).

Methods: The hsp60 gene was amplified by PCR, expressed in the vector pDEV-107 and transformed into Escherichia coli. The recombinant protein, expressed as a $\beta$-galactosidase fusion product, was captured onto a solid phase using a monoclonal antibody to $\beta$-galactosidase. A sensitive and specific ELISA to $C$ trachomatis hsp60 was produced. 113 patients with acute NGU were studied and of these, $89(79 \%)$ attended at least once for followup, 10-92 days after treatment, and were evaluable. A PCR assay and direct fluorescence antibody test were used to detect $C$ trachomatis at initial presentation and during follow-up. 
Results: Sixty-one (69\%) had evidence of chronic urethritis during follow-up. Detection of $C$ trachomatis at initial presentation or chlamydial antibody were not associated with the development of chronic urethritis. $C$ trachomatus was detected in only two patients during follow-up. Chlamydial hsp60 antibody was associated with the detection of $C$ trachomatis $(p<0.003)$ and chlamydial antibody ( $p<0.00003)$. Hsp60 antibody was not associated with the development of chronic urethritis in those patients who were chlamydia-positive $(p=0 \cdot 12)$. However, when all study patients were considered, irrespective of whether they were chlamydia-positive, hsp60 antibody was associated with the development of chronic urethritis $(\mathrm{p}<0.008)$.

Conclusions: The immune response to hsp60 is important in the pathogenesis of chronic NGU. The results also suggest that chronic NGU is not a consequence of continued production of hsp 60 by $C$ trachomatis.

\section{Hormonal factors and the detection of Chlamydia trachomatis in women}

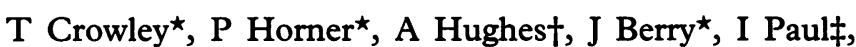
O Caulf. *Department of Genitourinary Medicine, Bristol Royal Infirmary, Bristol, †Department of Social Health, University of Bristol, and $¥$ Public Health Laboratory Service, Bristol

Aims: To examine whether there is a relationship between the menstrual cycle and the detection of Chlamydia trachomatis at the lower genital tract.

Methods: 1056 new and reregistered consecutive women attending a genitourinary medicine clinic requiring speculum examination were screened for $C$ trachomatis by enzyme immunoassay (IDEA, Dako Diagnostics Ltd.)

Results: Of 1022 women who had results available from both the cervix and the urethra, $C$ trachomatis was detected in $8.8 \%(89 / 1022)$ at any site, $2.3 \%(23 / 1022)$ at both sites, $7 \cdot 2 \%(74 / 1022)$ at the cervix alone and $1.5 \%(15 / 1022)$ at the urethra alone. Sampling at the urethra increased detection by $17 \%$ (15/89). Analysis of 808 women with a regular menstrual cycle showed a significant association of combined oral contraceptive use, age and ectropion with the detection of $C$ trachomatis. In addition the detection of $C$ trachomatis showed a significant variation with the menstrual cycle $(p=0.023)(R R$ 1.7 [95\% CI 1-2.8]). It was detected significantly more often in the latter part. This variation with the menstrual cycle was significant only in women who were combined oral contraceptive users $(\mathrm{p}=0.008)(\mathrm{rr}=2.3(1 \cdot 24-4 \cdot 5))$. Conclusion: Combined oral contraceptives, ectropion and youth are markers for the carriage of $C$ trachomatis in the lower genital tract of women. It is also detected significantly more often in the latter part of the menstrual cycle in women who are oral contraceptive users.

\section{Gonorrhoea in inner London: the hidden epidemic}

N Low ${ }^{1}$, B Molokwu'1, G Daker-White ${ }^{2}$, D Barlow ${ }^{2}$, A Pozniak'. 'Academic Unit, Department of Genitourinary Medicine, King's College School of Medicine and Dentistry, London. ${ }^{2}$ Department of Genitourinary Medicine, St Thomas' Hospital, London

Background: Rates of gonorrhoea in England and Wales are now below the Health of the Nation target of no more than 49 cases per 100000 population aged 15-64 years.
To see if this reflects improved sexual health for all we studied the distribution of gonorrhoea in three south London boroughs.

Methods: Episodes of gonorrhoea in adults resident in the postal areas of Lambeth, Southwark and Lewisham (LSL) in 1994 and 1995 were enumerated in 11 departments of genitourinary medicine and assigned to geographic wards. Standardised morbidity ratios (SMRs) for each of 73 wards were calculated by applying age- and sex-specific gonorrhoea rates of LSL as the reference population. Ecological associations between ward-specific SMRs, Jarman deprivation scores and ethnic composition were examined using Poisson regression models.

Results: 1978 non-repeat episodes of gonorrhoea, from 1 January 1994 to 31 December 1995, in residents of LSL were included. Rates were highest in 15-19 year old women, 580/100 000/year, and 20-24 year old men, $469 / 100000 /$ year and were 8 to 9 times higher in a combined non-white ethnic group than in whites in all age and sex strata. Evidence for geographical clustering was suggested by maps of ward-specific SMRs. Ward-specific SMRs were strongly positively associated with wardspecific deprivation scores $(r=0.52, p<0.0001)$ and with proportion of the ward population whose ethnic origin was not white $(r=0.65, p<0.0001)$. The association with non-white ethnicity persists after adjusting for socioeconomic deprivation.

Conclusions: Gonorrhoea rates in young adults in LSL rival those seen in poor urban areas in the USA. Low national rates mask an inner city epidemic which is associated with poverty and ethnicity at the population level There is an urgent need for further culturally appropriate research and intervention for gonorrhoea in inner London.

\section{Association of bacterial vaginosis with a history of second trimester miscarriage}

JM Llahi-Camp, R Rai, C Ison, L Regan, D TaylorRobinson. MRC/STD Research Group, Jefferis Wing, St Mary's Hospital, London

Objective: The aim of this study was to determine whether bacterial vaginosis (BV) is associated with a history of recurrent pregnancy loss.

Methods: 600 consecutive patients attending the Recurrent Miscarriage Clinic were screened for the presence of BV using a Gram-stained vaginal smear.

Results: Women who had had at least one late miscarriage had BV twice as commonly $(27 / 130 ; 21 \%)$ as women who had had only early losses $(31 / 370 ; 8 \%)$, p < $0.001\}$. The difference was even larger $(26 \%$ vs $8 \%)$ if women who had had term pregnancies were excluded. In addition, BV was found three times more commonly in Afro-Caribbean women $(17(29 \%)$ of 58$)$ than in Caucasian women $(36(9.4 \%)$ of 379$)$ and, in both ethnic groups, BV was diagnosed at least twice as frequently in those with a history of at least one late miscarriage than in those who had experienced first trimester pregnancy losses only $(p<0 \cdot 001)$. Furthermore, the condition occurred twice as often among smokers than non-smokers and, in both these groups, it was at least twice as common in women with a history of at least one late miscarriage as in those who had had early pregnancy losses only $\{p<$ $0.001\}$. However, the relation of BV to smoking was independent of ethnic origin. Women who douched with Dettol were mostly Afro-Caribbean and had BV more than twice as often as women who did not douche.

Conclusions: BV was associated significantly more with 
late miscarriages than with early losses in women with a history of recurrent miscarriage, irrespective of ethnic origin. It was more common in smokers and those who douched with Dettol.

\section{DELTA in the provinces-six months on}

JM Craig, KE Rogstad, GR Kinghorn. Department of Genitourinary Medicine, Royal Hallamshire Hospital, Sheffield

Aims: To assess impact of the preliminary results of the DELTA trial, six months after its release, on the management of HIV seropositive patients attending a provincial genitourinary department.

Method: Review of casenotes for all HIV seropositive patients regularly attending the Department of Genitourinary Medicine in Sheffield. Patients were considered eligible if they had an absolute CD4 count of 350 or less before 26 March 1996.

Results: At 3 months, 43 patients were eligible; mean age $35 \cdot 2$ years, mean CD4 count 214 (SD \pm 119 ) and 35 $(81 \%)$ were gay/bisexual men. 28 had prior antiretroviral therapy (ART), of these 20 were currently on treatment. 15 patients were therapy-naive. $18(42 \%)$ of eligible patients had their drug therapy altered to combination ART. Of non-naive patients, $15(54 \%)$ had a change in ART; 5 at patient's request and 10 as clinician's decision. Of naive patients, $3(25 \%)$ commenced ART; 1 at patient's request and 2 as clinician's decision. In total, 6 patients commenced or recommenced ART whilst 12 had treatment modifications. Reasons for not changing included patient's choice, fluctuating CD4 counts, already on combination therapy, advanced AIDS or potential drug interactions. Before the DELTA trial results, 20 patients were taking ART with 14 on monotherapy. 3 months later, 26 patients were on treatment with 23 on combination therapy $(78 \%$ taking AZT/ddC) and 3 choosing to remain on AZT alone. 4/23 have experienced intolerable side-effects, $3 / 4$ with AZT/ddI and $1 / 18$ with AZT/ddC. 1 patient on $\mathrm{AZT} / \mathrm{ddC}$ has been non-compliant. Data relating to outcome at six months and costs of these drug changes will be presented.

Conclusions: It was anticipated that a considerable number of patients attending our clinic would require additional antiretroviral therapy but 3 month outcome suggests that, despite initial patient interest, the actual uptake was lower than expected for a variety of reasons. As AZT/ddI combination therapy is poorly tolerated by our patients, AZT/ddC is at present our preferred choice of combination antiretroviral therapy.

\section{The HIV Partner Notification (PN) Project; an evaluation of HIV PN in England}

K Fenton, M Adler, J Giesecke, $S$ Trotter et al. The HIV PN Project and the UCL Department of STD
Background: In December 1992, the Department of Health (DoH), England, issued guidance on HIV partner notification. The HIV Partner Notification (PN) Project was a $\mathrm{DoH}$ funded evaluation of an active programme of HIV PN within GUM clinics in England.

Objectives: 1. To evaluate the effectiveness of a standardised PN procedure in reaching people unaware of their increased risk of HIV infection, 2. To determine factors which influence the process and outcomes of HIV $\mathrm{PN}$ in this setting, 3. To determine the resource requirements (health care worker training and time) required for PN.

Methods: Nineteen GUM clinics across England participated in the project. All newly diagnosed HIV positive patients seen at the participating centres within the one year study period were eligible for inclusion. Eligible patients were seen by health advisers who were responsible for collecting data from patients and their partners and co-ordinating PN activity. Information on existing HIV PN policies and practice within participating and a group of comparison clinics were also collected.

Results: Of 501 newly diagnosed HIV positive patients seen during the study period only $43 \%$ had documented evidence of PN being performed. The most common reason for not performing PN was the patient being assessed by a health care worker, as "too emotionally distressed" to do so. Seventy patients were recruited to the more detailed Project evaluation. They named 158 partners as being at risk of acquiring HIV, of whom 71 were notified. Among the contacts in whom notification was verified ( $n$ = 28), 27 were HIV tested and 5 new HIV positives were diagnosed. A number of factors (including acceptability, confidentiality concerns and patient mobility) which impacted negatively on the process of HIV PN within GUM clinics were revealed.

Conclusions: The Project found HIV PN to be successfully achieved in a minority of newly diagnosed patients, with a wide variation in clinic practices and policies for HIV PN. Arising from the project's recommendations, it is anticipated that new guidelines on HIV PN will be developed and appropriate training for health care workers involved in PN, explored.

\section{Sexual mixing: at home and abroad}

D Barlow, G Daker-White, B Band. St. Thomas' Hospital, Department of Genito-Urinary Medicine

Objective: To inform models of sexually transmitted disease epidemiology by describing the extent of assortative sexual mixing in a heterosexual population of GUM clinic attenders.

Methods: A linked serosurvey of HIV infection asked patients for the countries of birth (CoBs) of themselves, their parents and their sexual partners (in and outside of the UK) in the preceding twelve months. Patient's CoBs were compared with those of their sexual partners and with known locations of intercourse abroad. UK-borns were further stratified by Commission for Racial Equality

\begin{tabular}{|c|c|c|c|c|c|c|c|}
\hline \multirow[b]{2}{*}{ Patients } & \multicolumn{3}{|l|}{ Sex in the $U K$} & \multicolumn{4}{|l|}{ Sex outside the UK } \\
\hline & $\begin{array}{l}\text { No (\%) patients } \\
\text { reporting sex } \\
\text { in UK }\end{array}$ & $\begin{array}{l}\text { No (\%) known } \\
\text { partners with same } \\
\text { CoB as patient }\end{array}$ & $\begin{array}{l}\text { No (\%) } \\
\text { patients reporting } \\
\text { multiple partners }\end{array}$ & $\begin{array}{l}\text { No (\%) known } \\
\text { partners with same } \\
\text { CoB as patients }\end{array}$ & $\begin{array}{l}\text { No (\%) known } \\
\text { partners' CoBs = } \\
\text { country visited }\end{array}$ & $\begin{array}{l}\text { No (\%) visiting } \\
\text { Sub-Sah. Africa, } \\
\text { WI or Thailand }\end{array}$ & $\begin{array}{l}\text { No (\%) } \\
\text { patients reporting } \\
\text { multiple partners }\end{array}$ \\
\hline $\begin{array}{l}\text { UK born men, } \\
\text { CRE1 ( }=576) \\
\text { UK born men, } \\
\text { CRE2 ( }=122) \\
\text { UK born men, } \\
\text { CRE3 ( }=37 \text { ) }\end{array}$ & $\begin{array}{l}518 / 576 \\
(89 \cdot 9 \%) \\
119 / 122 \\
(97 \cdot 5 \%) \\
33 / 37 \\
(89 \cdot 2 \%)\end{array}$ & $\begin{array}{l}960 / 1172 \\
(81 \cdot 9 \%) \\
273 / 309 \\
(88 \cdot 4 \%) \\
58 / 67 \\
(86 \cdot 6 \%)\end{array}$ & $\begin{array}{l}293 / 518 \\
(56 \cdot 6 \%) \\
89 / 119 \\
(74 \cdot 8 \%) \\
20 / 33 \\
(60 \cdot 6 \%)\end{array}$ & $\begin{array}{l}196 / 872 \\
(22 \cdot 5 \%) \\
26 / 174 \\
(14 \cdot 9 \%) \\
3 / 77 \\
(3 \cdot 9 \%)\end{array}$ & $\begin{array}{l}336 / 743 \\
(45 \cdot 2 \%) \\
93 / 125 \\
(74 \cdot 4 \%) \\
53 / 84 \\
(63 \cdot 1 \%)\end{array}$ & $\begin{array}{l}57 / 420 \\
(13 \cdot 6 \%) \\
54 / 91 \\
(59 \cdot 3 \%) \\
21 / 31 \\
(67 \cdot 7 \%)\end{array}$ & $\begin{array}{l}163 / 562 \\
(29 \cdot 0 \%) \\
42 / 120 \\
(35 \cdot 0 \%) \\
18 / 37 \\
(48 \cdot 7 \%)\end{array}$ \\
\hline
\end{tabular}


(CRE) ethnic groups $(1=$ White, $2=$ Black Caribbean, 3 = Black African).

Results: Of 15878 respondents to the survey (1993-94), $1344 / 7451(18 \cdot 0 \%)$ men and $1030 / 8427(12 \cdot 2 \%)$ women reported sex abroad in the preceding 12 months. The table shows selected results in UK-born men. The following numbers of male respondents in each group in the table had had sex abroad: CRE1 (576/2948, 19.5\%), CRE2 (122/1410, 8.7\%), CRE3 (37/239, 15.5\%).

Conclusions: For all UK-born men, sex in the UK is significantly more assortative than sex outside the UK. CRE1 men are less likely to have UK-born partners in the UK than CRE2/3 men. A majority of UK-born CRE2/3 men who had sex outside the UK did so with partners born in the countries visited, which were also more likely to be those countries with higher heterosexual HIV prevalence. These sub-populations of clinic attenders may be at greater risk of acquiring HIV when abroad. However, more CRE1 men had had sex abroad. These results could assist the modelling of HIV transmission between nations with different seroprevalence rates.

\section{Recall responses to HPV16 by CD4 T cells from peripheral blood}

P Noble, P Beverley ${ }^{1}$. Academic Department of Sexually Transmitted Disease, 'Department of Immunology, University College London Medical School

Background: The immune response to human papillomavirus type 16 (HPV16) is of importance for the development of vaccines to prevent cervical carcinoma. Although there have been a few reports of $T$ helper responses to HPV16, these have required extended culture in vitro and may be primary rather than recall responses.

Objective: To detect HPV16 specific, CD4 recall responses in peripheral blood mononuclear cells (PBMCs) from individuals infected with HPV16.

Method: PBMCs from women infected with HPV16 were pulsed with tritiated thymidine five days after setting them up in culture with recombinant vaccinia virus containing HPV16 genetic material. Wild type virus and an irrelevant vaccinia construct were used as controls.

Results: PBMCs from twenty HPV16 positive patients and ten uncharacterised controls were screened. In three of the patients there was proliferation to one or more of the HPV16 constructs and virtually none to the wild type virus or the control construct. These findings are suggestive of HPV16-specific, recall proliferation. Extended culture of PBMCs from control individuals demonstrated generalised suppression of proliferation apparently mediated by HPV16 recombinant vaccinia.

Conclusions: We have developed a method to detect CD4 recall responses to HPV16 in the peripheral blood. That proliferation is sometimes suppressed in this system suggests a possible explanation for the difficulties others have reported in detecting such responses.

\section{Brighton HIV cross-sectional dermatology study}

S Uthayakumar, $\mathbf{R}$ Nandwani, $\mathrm{T}$ Drinkwater, AT Nayagam, CR Darley. ClaudeNicol Centre, Royal Sussex County Hospital, Brighton

Background: Cutaneous manifestations occur frequently in HIV infected patients. Previous studies have concentrated on single dermatoses and response to therapy.
Objectives: A prospective study to determine the prevalence and severity of skin problems and to elucidate the association with CD4 count in HIV+ patients attending a single department.

Methods: HIV+ patients who attended the study clinic over a period of 4 months were examined by the same dermatologist (CD). Prevalence of cutaneous signs were noted and demographic details, most recent CD4 count and CDC staging were cross referenced with clinic notes. A severity index was derived from the sum of skin problems.

Results: 151 patients were enrolled. The mean age was 38 years. 146 patients were male. The major risk factor was homo/bi sexuality (144). 37 patients had CD4 count < 200. 58 were asymptomatic and 35 had AIDS. 131 (91\%) patients had a skin disease. Total number of skin problems was 331. The most frequent problem was infection (170 occurrences in 102 patients), followed by dermatosis (124 occurrences in 97 patients), pruritus in 30 patients and malignancy in 7 patients. The most frequent diseases were tinea $(54 \%)$, eczema (46\%), viral infections $(26 \%)$ and dry skin $(23 \%)$. There were a significant association between both the number of skin problems and severity index and CD4 count $<200(p<0.0001)$ and with CDC stage IV (AIDS) $(\mathrm{p}<0.0001)$.

Conclusion: This is the largest prospective study undertaken in HIV+ patients looking at the skin manifestations only. Skin disease was present in $91 \%$ of patients. We have demonstrated statistically significant associations between CD4 count, CDC staging and both the presence and severity of skin diseases.

\section{An outbreak of multi-drug resistant tuberculosis (MDRTB) in a london teaching hospital HIVI genitourinary medicine unit: outbreak investigations, infection control issues and molecular epidemiology}

M Hannan, A Bell, P Easterbrook, M Nelson, B Gazzard, B Azadian, DA Hawkins. Departments of GUM/HIV and Microbiology, Chelsea and Westminster Hospital, London SW10

Objective: We report management and molecular epidemiology of the first nosocomial outbreak of MDRTb in a London HIV/GUM unit.

Method: The index case was an immigrant 33 year old Portuguese homosexual male with advanced HIV disease and a recent diagnosis of fully sensitive extra-pulmonary Mycobacterium tuberculosis on quadruple therapy. He had been admitted to an open HIV ward in April 1995 when we isolated $M$ tuberculosis resistant to INH, RIF, PYRZ, CLOF, RIFAB and ETHION from a liver biopsy. The patient died in June 1995. $168 \mathrm{HIV}$ positive patients, 60 health care workers and 57 community contacts were screened for $\mathrm{Tb}$ infection. We performed DNA fingerprinting with PCR and RFLP on all isolates from the index case, 7 other infected patients and $16 \mathrm{MDRTb}$ isolates from a Lisbon hospital.

Results: RFLP typing identified two genetically distinct strains of $M$ tuberculosis from the index case indicating that the patient had acquired a second infection with a multidrug resistant strain. Five patients who had shared the open ward and the two who had casual contact with the index case developed infection with this multi-drug resistant strain. Four of the 16 MDRTb strains from Lisbon were indistinguishable from our 8 strains of RFLP and PCR typing. No other contacts have developed the disease. Conclusion: The nosocomial dissemination of tuberculosis in an HIV/GUM unit is a major public health concern. 
A high rate of transmission of infection (up to $100 \%$ ) is seen in this susceptible population. The presentation of pulmonary tuberculosis in HIV patients may be atypical. Re-infection must also be considered. Molecular epidemiology reports from outbreaks in southern Europe suggest an increasing incidence ( $>30 \%$ ) of MDRTb in some hospitals. Infection control issues to reduce the likelihood of future nosocomial transmission will be discussed.

\section{Daily antiviral therapy for suppression of recurrent genital herpes in immunocompetent patients- current practice in the UK}

FM Cowan on behalf of the Herpes Simplex Advisory Panel. Department of Sexually Transmitted Diseases, University College London Medical School, London

Objectives: To determine the current practice of genitourinary medicine physicians with regard to prescribing daily antiviral treatment for the suppression of genital herpes.

Design: A self completion questionnaire was sent to one consultant in each department of genitourinary medicine in the UK ( $n=187)$.

Results: To date GUM physicians from 89 (49\%) departments have replied. Of these $66(74 \%)$ have some patients who receive daily antiviral therapy for suppressing genital herpes (median patients/department 5; range 0-120). In $40 \%$ of departments some patients receive suppressive therapy directly from their GP (median patients/department 3; range 1-32). In $41 \%$ of cases this is due to funding constraints within the department. Among departments which prescribe suppressive therapy, indications for treatment include frequently recurring genital herpes (100\%), psychological dysfunction (82\%), to decrease transmission to their longterm $(19 \%)$ or new $(27 \%)$ sexual partner, to prevent vertical transmission $(23 \%)$ or to prevent delivery by caesarian section (24\%). Department's definition of frequently recurring herpes vary (median 6; range 1-24 recurrences/year). Most departments (57\%) start treatment with aciclovir $400 \mathrm{mg}$ bd for six months or more (66\%). Where departments do not prescribe suppressive therapy they prescribe oral antiviral treatment for each recurrence. Only three departments use topical therapy. $63 \%$ of departments inform patients that they may be infectious while on treatment, while $22 \%$ do not discuss transmission risk. Centres which have taken part in trials of antiviral suppression do not appear to be more likely to prescribe treatment. (OR $2 \cdot 4 ; 95 \%$ CI $0 \cdot 6-9 \cdot 6$ ).

Conclusion: There is considerable variation in patients access to suppressive antiviral therapy across the UK. Although antivirals have not been shown to reduce the risk of either sexual or vertical transmission, daily antiviral treatment is given for these indications in a quarter of centres.

\section{Prevalence of sexual dysfunction in heterosexual patients attending a Central London Genitourinary Medicine (GUM) clinic}

D Goldmeier ${ }^{1}$, A Renton ${ }^{2}$, FEA Keane ${ }^{1}$, P Carter ${ }^{1} .{ }^{1}$ The Jefferiss Wing, ${ }^{2}$ The Academic Department of Public Health, St Mary's Hospital, Paddington, London

Background: The Golombok Rust Inventory of Sexual Satisfaction (GRISS) is a short questionnaire-based instrument designed to profile an individual's sexual func- tioning. The instrument generates a total score allowing identification of overall dysfunction together with subscale scores which detect dysfunction in specific clinical areas such as vaginismus and anorgasmia in women and erectile dysfunction and premature ejaculation in men. We employed this device to assess the prevalence of sexual dysfunction in patients attending the routine GUM clinic at the Jefferiss Wing, St Mary's Hospital, Paddington in London.

Method: Two hundred and thirty three new heterosexual attenders at the routine GUM clinic were invited to participate in this study, of whom 106 men and 105 women consented. Each subject completed a GRISS questionnaire and was asked additional information regarding sexual debut and behaviour as well as a previous history of sexual assault. The KC60 classification was used to record the STD diagnosis for each patient.

Results: A total GRISS score indicating overall sexual dysfunction was obtained for $25(24 \%)$ of men and 10 $(9 \%)$ of women, the prevalence being significantly lower in women $\left(p=0.01, \chi^{2}=6.56,1 \mathrm{df}\right)$. Sixty three men $(59 \%)$ and 63 women (60\%) produced scores indicative of significant abnormality on at least one sub-scaleincluding for erectile dysfunction-20 (19\%), premature ejaculation $-23(22 \%)$, vaginismus $-26(25 \%)$ and anorgasmia-23 (22\%). Of the 96 men and 93 women who replied, $8(8 \%)$ and $20(21 \%)$ respectively reported having been sexually assaulted previously. Neither an abnormal overall or sub-scale score on the GRISS was associated with a current STD on KC60 diagnosis or a history of sexual assault for either men or women.

Conclusion: There is a substantial prevalence of sexual dysfunction in new heterosexual attenders at our routine GUM clinic.

\section{Non-cultural detection of rectal and pharyngeal gonorrhoea}

H Young, J Anderson, A Moyes, A McMillan'. Scottish Neisseria gonorrhoeae Reference Laboratory, and 'Department of Genito-Urinary Medicine, Edinburgh Royal Infirmary

Many laboratories have difficulty in culturing Neisseria gonorrhoeae from rectal and pharyngeal sites. The objective of this study was to assess the sensitivity and specificity of the GEN-PROBER ${ }^{R}$ PACE $^{R} 2$ System for the non-cultural detection of rectal and pharyngeal gonorrhoea. Duplicate rectal and pharyngeal swabs were collected from eighty five gay men attending the Department of Genito-Urinary Medicine during the latter half of 1995. The first swab from each site was plated directly on to modified New York City medium. After incubation, oxidase positive Gram negative diplococci were confirmed as $N$ gonorrhoeae by both immunological and biochemical tests: non-gonococcal neisseriae were speciated on the basis of their carbohydrate utilisation pattern. The second specimen was collected using the GEN-PROBE ${ }^{\mathrm{R}} \mathrm{PACE}^{\mathrm{R}} 2$ collection swab and transport tube and stored at $-20^{\circ} \mathrm{C}$ before testing in batches. The GEN-PROBE ${ }^{\mathrm{R}} \mathrm{PACE}^{\mathrm{R}} 2$ System uses a chemiluminescent labelled single-stranded DNA probe that is complementary to the ribosomal RNA (rRNA) of the gonococcus: rRNA released from gonococci in the specimen combines with the labelled probe to form a stable DNA:RNA hybrid which is measured in a luminometer. Fourteen of the 85 patients $(16.5 \%)$ gave 18 positive gonococcal cultures (4 rectum and throat; 6 rectum only; and 4 throat only). GEN-PROBE was positive at 23 sites 
in 17 patients ( 6 rectum and throat; 5 rectum only; and 6 throat only). GEN-PROBE was negative on two throat specimens (one GEN-PROBE positive on repeat testing) and positive on six negative gonococcal throat cultures (four with meningococci): three of these patients had a positive gonococcal culture from the rectum and one was a known contact of gonorrhoea. One patient with culture positive urethral gonorrhoea had a positive GEN-PROBE result but a negative culture on a rectal swab. Throat swabs from 38 patients yielded non-gonococcal neisseriae, mainly meningococci, and gave negative GEN-PROBE results confirming high specificity of the test.

These results suggest that the GEN-PROBE PACE 2 System is more sensitive than culture for the detection of pharyngeal gonorrhoea.

\section{Sustained benefits after four years of saquinavir monotherapy}

DR Churchill, AS Pym, C Loveday, I Duncan, JN Weber. St Mary's Hospital Medical School, London; UCLMS; Roche Products Ltd

Objective: To describe clinical progression and changes in surrogate markers in patients on saquinavir monotherapy over four years.

Methods: 49 HIV-infected patients entered a single-centre phase I/II study of saquinavir between August 1991 and August 1992. Patients were drug-naive, asymptomatic or minimally symptomatic, and had CD4 counts below $500 \times 10^{6} / 1.15$ patients continued on long-term saquinavir $600 \mathrm{mg}$ tid as monotherapy for a median of 46 months (range 36-49). Patients were evaluated clinically, and by changes in CD4 count, plasma viraemia, and point mutations in the viral protease gene.

Results: Of the 15 patients on long-term saquinavir, 2 patients developed an AIDS diagnosis during follow-up. Median CD4 count fall from baseline was 80 cells (16\%) over a median period of 46 months. Median viral load increase from baseline was $0.67 \mathrm{log} .50 \%$ of patients so far studied had an L90M mutation in the protease gene at 17-35 months. In some individuals, viral load and CD4 changes have been minimal, despite the emergence of the L90M mutation.
Conclusions: No other group of patients worldwide have received monotherapy with any protease inhibitor for such an extended period. These results suggest slow progression of HIV disease in these patients. Although this may be in part due to selection bias, the possibility is raised that long term saquinavir therapy is of sustained clinical benefit, despite evidence of genotypic resistance.

Fluctuations of human immunodeficiency virus in the semen of HIV-infected men with newly acquired sexually transmitted diseases

EM Carlin 1 , MC Atkins ${ }^{2}$ FC Boag', VC Emery ${ }^{2}$, PD Griffiths $^{2}$. 'John Hunter Clinic, Chelsea and Westminster Hospital, London. ${ }^{2}$ Department of Virology, Royal Free Hospital School of Medicine, London

Objectives: To quantify the human immunodeficiency virus (HIV) load in the semen of HIV-infected men presenting with acute sexually transmitted diseases (STDs) and to determine if viral load decreases in serial semen samples when the acute infection is treated.

Methods: HIV seropositive men presenting with microscopically confirmed acute urethritis were recruited to the study. Blood and semen samples were collected at presentation before treatment was given and at subsequent visits after antibiotic therapy. The samples were analysed using the polymerase chain reaction (PCR) to identify proviral DNA and positive samples were quantified using a competitive PCR.

Results: Three men had culture-confirmed gonococcal urethritis, one complicated by post-gonococcal urethritis. The fourth man had non-gonococcal urethritis. Appropriate antibiotic therapy was given. The HIV proviral load in the semen of all four men declined when their intercurrent STDs were treated $(p<0.05$, MannWhitney test), whereas there were no significant changes in the blood.

Conclusions: The presence of HIV in the semen has been well described and sexual transmission is the most common mode of virus acquisition. Our results help explain how transmission of HIV may be facilitated by concomitant STDs and add further support for an aggressive approach to treating STDs as a means of reducing HIV infection. 